\title{
An analysis of visual adaptation and contrast perception for Tone Mapping
}

\author{
Sira Ferradans, Marcelo Bertalmío, Edoardo Provenzi and Vicent Caselles
}

\begin{abstract}
Tone Mapping is the problem of compressing the range of a High Dynamic Range image so that it can be displayed in a Low Dynamic Range screen, without losing or introducing novel details: the final image should produce in the observer a sensation as close as possible to the perception produced by the real world scene. We propose a tone mapping operator with two stages. The first stage is a global method that implements visual adaptation, based on experiments on human perception, in particular we point out the importance of cone saturation. The second stage performs local contrast enhancement, based on a variational model inspired by color vision phenomenology. We evaluate this method with a metric validated by psychophysical experiments, and in terms of this metric our method compares very well with the state of the art.
\end{abstract}

\section{INTRODUCTION}

High Dynamic Range (HDR) images constitute a powerful tool to store real-world radiance values and have become more and more accessible during the last fifteen years. However, radiances may span many orders of magnitude, while common displays and printers usually span up to two [1]. So, a further 'compression step', called 'Tone Mapping' (TM) is required to properly visualize the information stored in HDR images. Many tone mapping operators (TMOs) have been proposed in the literature; for a thorough review and analysis of the state of the art until 2005, we refer to the excellent book by Reinhard et al. [2]. Here we just want to give a very brief overview of the different schools of thought that have been proposed so far.

There is a category of perceptually-based TMOs which can be either spatially global or local. The former are, in general, very fast and do not introduce halos or artifacts, but their contrast rendition tends to be poor. The first global TMOs used Stevens' law [3] to achieve range compression and were proposed by Tumblin et al. [4], Chiu et al. [5] and Ward et al. [6]. Schlick [7] proposed a rational function very close to the Naka-Rushton formula (that will be discussed in detail in next section) showing improvements with respect to Stevens' law. The global Naka-Rushton formula has been exploited by Pattanaik et al. [8], Reinhard and Devlin [9], and more recently, by Kuang et al. [10]. More sophisticated vision models were also proposed, taking into account timeadaptation such as Ferwerda's model [11] or Weber-Fechner's law, e.g. the methods by Ward et al. [12] and Ashikhmin et al. [13]. Reinhard et al. [14] and Tamburrino et al. [15] introduced local TMOs that improved detail but created halos and artifacts

e-mail: $\quad$ sira.ferradans, marcelo.bertalmio, edoardo.provenzi,vicent.caselles $\} @$ upf.edu. Tel. +34 93542 2937. Fax. +34 93542 2569. Departamento de Tecnologías de la Información y las Comunicaciones, Universitat Pompeu Fabra, c/ Tànger 122-140, 08018, Barcelona, Spain. next to edges. A TMO based on a modified version of the Retinex model of color vision was proposed by Jobson et al. [16], and the anchoring theory 1 has been used by Krawczyk et al. [17] after a suitable subdivision of the original image into layers of similar luminance.

There is another category, that of gradient-based TMOs, which rely on the idea of shrinking large intensity gradients while preserving small fluctuations, corresponding to fine details. Tumblin et al. [18] used a hierarchical method based on a Partial Differential Equation (PDE) inspired by anisotropic diffusion. Durand et al. [19] and Kuang et al. [10] obtained improved results by using techniques inspired by bilateral filtering. Fattal et al. [20] and Mantiuk et al. [21] proposed methods where spatially varying compression factors were used to implement suitable manipulations of the gradient field.

Finally, Pardo et al. [22] and Lischinski et al. [23] proposed interactive TMOs in order to produce pleasant results for particular luminance zones selected by a user.

In this paper we will follow the TM philosophy proposed by Ward et al. [12] which is that the main purpose of TM is to emulate as much as possible the perception of contrast and color produced by the real-world scene. Lacking a perfect model of the Human Visual System (HVS), we concentrate instead on well established facts of visual perception (experimental data on visual adaptation and contrast perception) to propose our TM operator.

Dunn et al. [24] refer to visual adaptation as a completely retinal process, while local contrast enhancement (and color constancy) also involves the visual cortex (see Hubel's book [25]). This suggests that the processes involved in these two phases are quite different, therefore we have decided to mimic them (or rather their outputs) in two independent stages of our algorithm.

The main contribution of this article is to propose a TMO which produces good results by trying to replicate basic properties of the HVS. At the same time, we point out and make use of several aspects of the HVS, supported by psycho-physiological data, that we feel have been ignored in the TM literature and which are influential in the quality of the TM results. Some of these aspects are, for example, taking into account localized cone saturation, or computing the semisaturation constant in the Naka-Rushton equation as an average of each chromatic channel, but not of the luminance.

\section{VISUAL ADAPTATION}

Let us begin this section by recalling how the retina responds to light stimuli. The range of radiances over which

\footnotetext{
${ }^{1}$ The anchoring theory is best known in the image processing literature as encompassing white-patch or gray-world methods.
} 
the HVS can operate is very large, from $10^{-6} \mathrm{~cd} / \mathrm{m}^{2}$ (scotopic limit) to $10^{6} \mathrm{~cd} / \mathrm{m}^{2}$ (glare limit): the automatic process that allows the HVS to operate over such a huge range is called visual adaptation. It is important to stress that the HVS cannot operate over its entire range simultaneously. Rather, photoreceptors adapt locally to the average of nearby retinal illumination values (depending on photopigment bleaching and gaze direction) so that the HVS handles a smaller magnitude interval. There is no complete agreement in the literature about the precise value of this range, which can vary in orders of magnitude from two according to Shapley and Enroth [26] (pg.326) to four according to Keener and Sneyd [27] (pg.670): estimates vary widely due to asymptotic responses to contrast extremes.

Neuroscience experiments to measure visual adaptation have been performed using very simple, non-natural images: on a uniform background were superimposed brief pulses of light with intensity $\mathcal{I}$. When a photoreceptor absorbs $\mathcal{I}$, the electric potential of its membrane changes accordingly to the empirical law known as the Michaelis-Menten equation (after Valeton and van Norren [28]):

$$
r(\mathcal{I}) \equiv \frac{\Delta V(\mathcal{I})}{\Delta V_{\max }}=\frac{\mathcal{I}^{n}}{\mathcal{I}^{n}+\mathcal{I}_{s}^{n}},
$$

or as the Naka-Rushton equation when $n=1$ (see Shapley and Enroth [26]). In this equation, $\mathcal{I}_{s}$ is the light level at which the photoreceptor response is half maximal, called semisaturation level and which is usually associated with the level of adaptation. Each type of cone is most sensitive over a particular waveband, thus the semisaturation constant must depend on the amount of light in this same waveband that reaches it, not on the luminance of the light source, as Boynton [29] and Wade and Wandell [30] point out. The change of electric potential $\Delta V(\mathcal{I})$ is the photoreceptor's physiological response to $\mathcal{I}, \Delta V_{\max }$ is the highest difference of potential that can be generated, and $n$ is a constant (e.g. Valeton and van Norren [28] give $n=0.74$ for the rhesus monkey). We should point out that this change in electric potential $\Delta V(\mathcal{I})$ describes the light response of one single living photoreceptor isolated in a tiny glass pipette, and it is not the response of the cell embedded in the retina. Adjacent photoreceptors strongly affect each other both chemically and electrically: none of the retina's approximately 125 million photoreceptor cells respond independently, and the brain receives no lightmeter-like signals from them. Instead, three layers of retinal neurons interconnect to construct 20 or more different kinds of signals that summarize local changes in retinal illumination (well-described by 'receptive field' models, see Palmer [31]). Little more than 1 million retinal ganglion cells (the innermost retinal layer) send these change-summaries towards the brain, by varying their firing rates $(1-100 \mathrm{~Hz})$. A bundle of their extremely long $(2-3 \mathrm{~cm})$ individual axons comprise the optic nerve bundle that leaves the back of each eyeball, even including response signals traveling from the brain back to the retina.

Eq. (1) has been used extensively in the TM literature, as mentioned in the previous section: Pattanaik et al. [8], Reinhard and Devlin [9] or Tamburrino et al. [15] all use a modified version of eq. (1), apply it to an HDR image with values $\mathcal{I}$, and obtain as an output $r(\mathcal{I})$. Although it has not been explicitly stated in the TM literature, the underlying assumption here is that the output of eq. (11), a ratio of voltage amplitudes of electrical responses (or the ratio of light intensities), a physical magnitude, is assumed to be correlated with the perceived brightness, which is a sensation, a perceptual magnitude.

We will come back to this later, let us just recap by saying that TMOs which use eq. (1), or variations of it, take an HDR input intensity $\mathcal{I}$ and give an LDR output $r(\mathcal{I})$ trying to mimic the perceived brightness.

Regarding perception, the foundational experiments of psychophysics were developed in the mid-nineteenth century, by the German physician E.H. Weber. His experiments with handheld weights showed ratios predicted detection thresholds for many stimuli. These rules agreed fairly well with later visual experiments by others where, with a similar set-up as the ones described above (increments of light on a uniform background), instead of measuring the electric response inside the retina the subject was asked when the difference between the background light $\mathcal{I}$ and the superimposed light $\mathcal{I}+\Delta \mathcal{I}$ was noticeable. The minimum difference $\Delta \mathcal{I}$ which the subject is able to perceive is called the Just Noticeable Difference (JND). It was found that the ratio between the JND and the background intensity $\mathcal{I}$ is constant for a wide range of values of $\mathcal{I}$, which is expressed in what is known as Weber's law:

$$
\frac{\Delta \mathcal{I}}{\mathcal{I}}=k,
$$

where $k>0$ is a perceptual constant called Weber fraction.

In $\log$-log units, the relationship between $\Delta \mathcal{I}$ and $\mathcal{I}$ is linear, with slope 1: $\log (\Delta \mathcal{I})=\log (\mathcal{I})+\log (k)$. However, Weber's Law does not hold for low intensity values, where experiments show that the slope tends to zero. To account for this, Weber's colleague G. Fechner introduced the concept of 'dark light', defining what is now known as the Weber-Fechner's law:

$$
\frac{\Delta \mathcal{I}}{\mathcal{I}+m}=k,
$$

where $m>0$ is a quantity often interpreted as internal noise in the visual mechanism. Now, when $\mathcal{I}>>m$ the slope of $\log (\mathrm{JND})$ as a function $\operatorname{of} \log (\mathcal{I})$ is 1 ; but when $\mathcal{I}<<m$ the slope is close to zero and the curve matches the experimental data also at low intensity values. It is common to plot this JND vs. $\mathcal{I}$ data in $\log -\log$ scale in what are known as threshold versus radiance (t.v.r.) or threshold versus intensity (t.v.i.) curves. Performing these contrast sensitivity experiments using different colors of light for the background and the superimposed stimuli is called the two-color threshold method according to Wyszecki and Stiles [32] (pg. 527) and allows us to see that although the three types of cones have responses which follow Weber-Fechner's law, each type of cone has a different value for the Weber fraction $k$ in eq. (3).

When the conditioning stimuli and background are static (i.e. the retina has adapted fully to them) the t.v.r. curves for the three types of cones extend indefinitely, following eq. (3) from Wyszecki and Stiles [32] (pg. 547). This fact 
is usually stated in the TM literature (e.g. in [11], [33]) as "the cone system never saturates", but this statement might be misleading: Wyszecki and Stiles [32] (pages. 547-8) show that the cone responses can saturate, only not with a static field in these experiments, but with a pulsated one (brief intense conditioning stimuli). Also, cone saturation does not require pulsed light: any static HDR scene will cause transient, localized cone saturation each time we re-direct our gaze. Fig. 1. adapted from Shevell [34], shows two different t.v.r. curves for the same subject and same type of photoreceptor ("Red" cone). When the stimuli field is steady, the t.v.r. is the lower one, which follows eq. (3) closely. However, when the field is pulsed, the t.v.r. is the upper one, which increases very steeply after a certain point, implying that the cones are saturated for higher radiances. The saturation of the cones is a key element of our approach and we will return to it later.

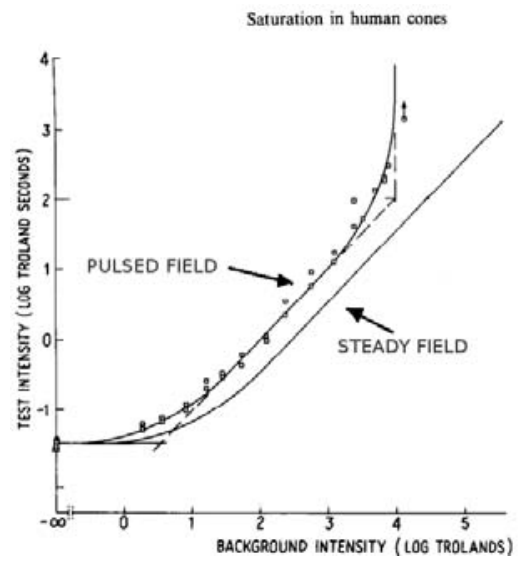

Figure 1. Cones saturate when the stimuli field is not steady but pulsed: perceptual t.v.r. curves of cones for pulsed and steady stimuli, figure adapted from Shevell [34].

We now follow the approach of Wyszecki and Stiles [32] (pg. 490) and postulate that the JND can be used as a "sensation magnitude" because it corresponds to (minimum) equal increments of sensation. This allows us to rewrite WeberFechner's law in the following way:

$$
\frac{\Delta \mathcal{I}}{\mathcal{I}+m}=k \Delta s,
$$

where $\Delta s$ is the increment in the sensation-magnitude function $s(\mathcal{I})$, also called "perceived brightness". Integrating eq. (4) we obtain:

$$
s(\mathcal{I})=\hat{k} \log (\mathcal{I}+m)+s_{0},
$$

where $\hat{k}=\frac{1}{k}$. This equation is a simple logarithmic mapping and it has also been used extensively in TM, where for an input intensity $\mathcal{I}$ the output is the perceived brightness $s(\mathcal{I})$. As Reinhard et al. point out [2], this is probably the simplest procedure one might use as a TMO, and it works fairly well for medium-dynamic-range images. Nonetheless, this equation is questionable and still contested in the psychophysics community, where researchers disagree on the validity of accumulating and/or integrating JNDs to assess supra-threshold stimuli of any sort. S.S. Stevens [35] strongly challenged this practice and argued for power-law rules. But Stevens provoked vigorous, heated debate, and some works such as Macmillan et al. [36] question the validity of his experiments, so there does not seem to be definitive answers in this regard.

Now we have two equations, eq. (1) and eq. (5), which are different but which are used (directly or with some modifications) under the assumption that they are both modeling the same thing, namely the perceived brightness. This obviously cannot be, so let us look at this matter in more detail.

Naka-Rushton (NR) and Weber-Fechner (WF) equations certainly do not match, in fact the two equations mathematically model results of markedly different experiments that measured very different visual phenomena. WF describes detectable-difference thresholds for small, steady-state stimuli; users adapt to a steady, uniform background, and experiments measure the tiniest steady difference we can distinguish from that background. NR models electrical responses to flashed stimuli; it measures how well we can detect tiny differences in intensities far away from that of the adapting background (and measured before our adaptation level can change). Since NR does not match with WF, we cannot claim that a TMO based on the NR equation accurately models perception in the HVS. But this would seem to be only an issue from a theoretical point of view, because the TM results obtained with these NR-like methods, based on modifications of eq. (1), are quite good and usually better than those obtained with the logarithmic mapping of eq. (5), their overall appearance seems closer to our own perception. To try to understand this, let us recall that TMOs map an arbitrarily large radiance range into image values in a limited range, say $\{0, \ldots, 255\}$, but without loss of generality we may assume that the output range is the interval $[0,1]$. The values given by eq. (1) are already in this interval, but for eq. (5) it is necessary to apply a normalization step to the output. As we can see in Fig. 2, the outputs of eqs. (11) and (5) are fairly similar for the first three or four orders of $\mathcal{I}$, but then the output of eq. (1) tends to 1 while the output of eq. (5) increases indefinitely: after normalization, the final response curve is shown in the figure with the title "Scaled Weber-Fechner".

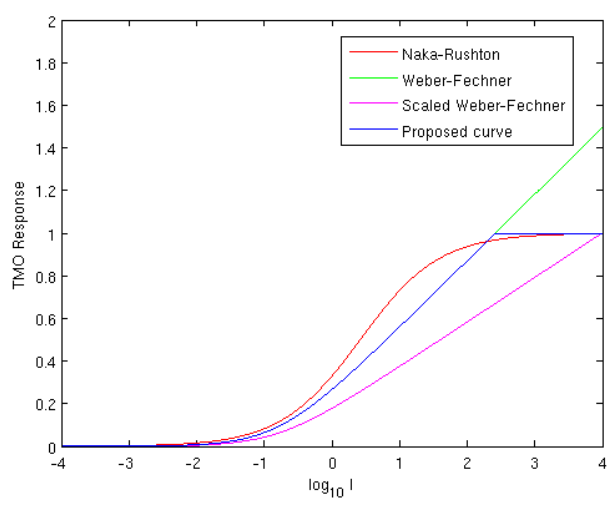

Figure 2. Tone mapping curves for the logarithmic mapping (Weber-Fechner law), the Naka-Rushton equation and the curve for the first stage of proposed method. 
The fact that eq. (1) tends to 1 for high radiance values is explained by the saturation property of photoreceptors: the activity of cones covers approximately four orders of magnitude around the semi-saturation value $\mathcal{I}_{s}$, as it is explained by Valeton and van Norren [28], and for higher radiances the cones saturate. The saturation of the cones is not consideredd by the Weber-Fechner law, and therefore the curve for eq. (5) always increases.

Taking all the above into account, we propose the following: since cones are indeed saturating, in order to model the perceptual response we must not use the t.v.r. curve obtained with steady stimuli fields, because these fields do not cause saturation in the cones and we end up with the logarithmic curve of eq. (5), which increases indefinitely. Instead, we must use the t.v.r. curve obtained with pulsating fields which, as it is shown in Fig. 11 complies with Weber-Fechner in an operative range of four orders of magnitude, but for higher radiances increases very steeply because the cones saturate at those radiance values. Dunn et al. [24], while talking about visual adaptation and saccades (voluntary or involuntary rapid movement of the eye), say the following: "retinal mechanisms [...] adjust sensitivity in the $200 \mathrm{~ms}$ intervals between saccades", and "As we make saccades to explore a visual scene, retinal neurons encounter a wide range of light intensities. Receptor and post-receptor adaptation permit the amplification required to see objects in shadows while avoiding saturation from the sky. The combination of these adaptive mechanisms allows the visual system to encode details in a scene with greater fidelity than a standard camera at a single exposure setting”. The implication would be that while viewing real-world scenes, as we frequently use saccadic movements to look for and track objects, or simply to gather all the details, the stimuli fields are not steady but pulsed. Hence the t.v.r. curve that more accurately matches perception in real-world images would be the upper curve in Fig. 1 .

In short, from Fig. 1 there seems to be an inflexion point at the radiance value located approximately two orders of magnitude above the semi-saturation radiance $\mathcal{I}_{s}$, which we will call $\mathcal{I}_{M}$ : for radiances below $\mathcal{I}_{M}$, Weber-Fechner's law holds so the perceptually valid tone mapping curve is the one given by eq. (5); for radiances above $\mathcal{I}_{M}$, the cones' responses start to saturate so the perceptually (and physiologically) valid tone mapping curve must have an horizontal asymptote, and for this we can use the curve given by the Naka-Rushton equation, eq. (1). With this information, we can now introduce the first stage of our algorithm. We propose to use a TM curve $c(\mathcal{I})$ which combines the curves for eqs. (5) and (1) in the aforementioned way:

$$
c(\mathcal{I})= \begin{cases}\hat{k} \log (\mathcal{I}+m)+s_{0}, & \mathcal{I} \leq \mathcal{I}_{M} \\ \frac{\mathcal{I}^{n}}{\mathcal{I}^{n}+\mathcal{I}_{s}^{n}}, & \mathcal{I}>\mathcal{I}_{M},\end{cases}
$$

where $s_{0}$ is chosen so as to ensure the continuity of $c$ at $\mathcal{I}_{M}$; the output of $c$ will be normalized, i.e. linearly scaled so that it spans the interval $[0,1]$. Fig. 2 depicts the curve associated with this equation. The implementation details will be given in section IV, but for the moment let us see an example. Fig. 3 shows the TM results obtained using eqs. (1), (5) and (6) on the same HDR image (the choice of parameters is the same as the one explained in section IV). The left-hand image, obtained using eq. (1), has an overall good contrast but the brightest regions appear overexposed (e.g. the areas nearby the windows or the trees); the middle image, obtained with the logarithmic mapping of eq. (5), has an overall poor contrast but the details of the brightest regions are not lost. The right-hand result is the one obtained with the first stage of our proposed method, eq. (6), and we see that it combines the best characteristics of the other two images, showing an overall good contrast and little or no loss of detail in the brightest areas.

\section{LOCAL CONTRAST ENHANCEMENT}

The first stage of our algorithm is a global TM method that uses the mapping curve $c$ defined in eq. (6) so as to be compatible with perceptual data. Since this curve is nondecreasing, the ordering of the level-lines (isocontours) of the HDR image is preserved, thus contrast is modified globally in a spatially-invariant way. However, Hubel, among others, has shown the importance that spatially-variant operators have in the HVS and how "differences in the spectral content of light across the visual field are necessary to perceive color" [25] (chapter 8). Spatial information allows the HVS to partially discard the influence of the illuminant when perceiving the colors of a given scene, a property known as human color constancy as Land has pointed out [37]. Moreover, it allows us to locally enhance details, a phenomenon observed in simple experiments such as the Mach bands effect. Thus, if the purpose of a TMO is to reproduce the color and contrast perception of a viewer in the given scene, these two features of the HVS should be taken into account, in the authors' opinion.

Bertalmío et al. [38] proposed a perceptually-inspired color enhancement algorithm that performs local contrast enhancement and approximates color constancy. Given an image $I_{0}$ to enhance, they define an energy functional $E(I)$ with three terms, one for local contrast $C_{w}(I)$, where $w$ is a weight function that will be specified later, one for dispersion around the average value $D(I)$ and one for attachment to the original image $I_{0}, A\left(I, I_{0}\right)$ :

$$
E(I)=-\alpha C_{w}(I)+\beta D(I)+\gamma A\left(I, I_{0}\right), \alpha, \beta, \gamma \in \Re^{+} .
$$

Please note that $I$ denotes the image function $I: \Omega \rightarrow \mathbb{R}^{+}, \Omega$ being the image domain, whereas in the previous section we used the calligraphic font $\mathcal{I}$ to denote a radiance value, i.e. a real number.

The contrast term $C_{w}(I)$ in eq. (7) measures a weighted average contrast over the image. The dispersion term $D(I)$ measures the average departure of the values of the pixels in $I$ from a given middle value (in Bertalmío et al. [38] this value is 0.5$)$. The attachment term $A\left(I, I_{0}\right)$ measures the average departure of the values of the pixels in $I$ from their corresponding values in $I_{0}$. Since $\alpha, \beta, \gamma>0$, the minimization of $E$ produces an image $\tilde{I}$ where the contrast has been locally enhanced (as $-\alpha<0$, maximizing $C$ contributes to minimizing $E$ ) but without departing too much from either the middle value or the original data.

A generalization of this perceptually-inspired variational method was given by Palma-Amestoy et al. [39], where the 

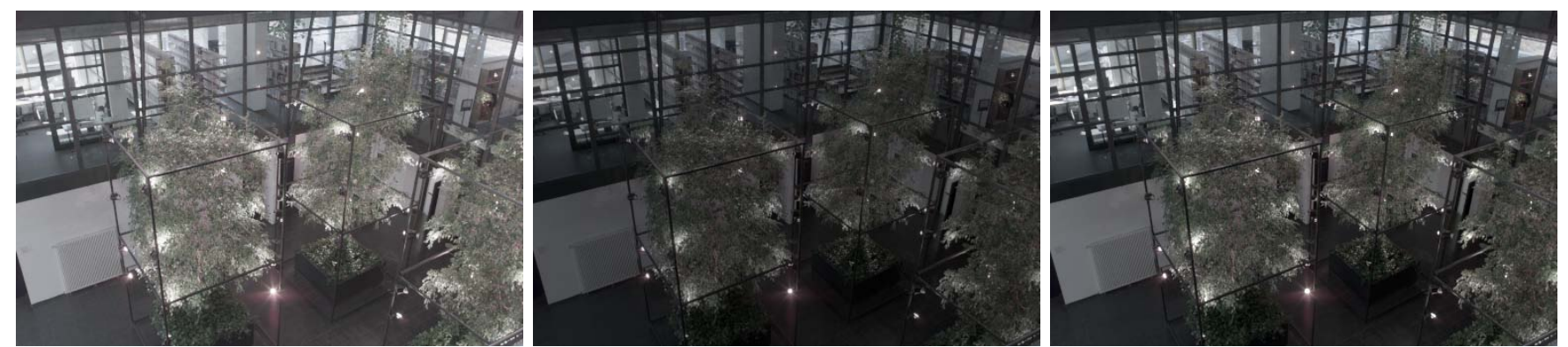

Figure 3. Left: TM output using the Naka-Rushton equation. Middle: TM output using a logarithmic mapping (Weber-Fechner's law.) Right: TM output of the first stage of our method, eq. 6.

authors suggest a set of basic requirements to be fulfilled by an energy functional so that it can be considered as "perceptually inspired" (namely color constancy, local contrast enhancement and gray-world mechanism) and prove that there is only one class of functionals satisfying all of these requirements.

Bertalmío et al. introduced [40] a variational implementation of the Retinex theory proposed by Land [37] called Kernel-Based Retinex (KBR). The original Retinex theory aims at reproducing the sensory response of the HVS to color stimuli: it can reduce the effect of color cast and enhance details in low-key images but, since it can only increase pixel intensities, it is not able to enhance over-exposed pictures [41]. Modifying the symmetry in the functional that describes Retinex, the authors obtain a variational method belonging to the family of the ones proposed by Palma-Amestoy et al. [39]. Therefore, we can establish a close relationship between the functional in eq. (7) and Retinex theory, which in turn was based on perceptual experiments.

Taking all this into account, we propose as the second step of our TMO to find the image $\tilde{I}$ that minimizes this expression:

$$
\begin{array}{r}
E(I)=-\frac{1}{4} \iint_{\Omega^{2}} w(x, y)|I(x)-I(y)| d x d y+ \\
\int_{\Omega}(I(x)-\mu)^{2} d x+\int_{\Omega}\left(I(x)-I_{0}(x)\right)^{2} d x,
\end{array}
$$

where we have chosen specific values for the constants $\alpha, \beta$ and $\gamma$, the Gaussian kernel $w$ gives locality to the contrast measure (because $w(x, y)$ decreases with the distance $\|x-y\|$ ), and $\mu$ is the average value of the original image $I_{0}$. The image $I_{0}$ is the (normalized) output of the first stage of our TMO, i.e. given an input HDR image we apply eq. (6) to it and obtain the LDR image $I_{0}$. The functional in eq. (8) is a slight modification of the one proposed by Bertalmío et al. [38]: we use the actual average $\mu$ instead of 0.5 for the dispersion term, and the absolute value instead of a smoothed version of it in the contrast term.

The final output of our TM algorithm is then the image $\tilde{I}$ minimizing eq. (8). The details of this minimization process will be given in the following section, but for the moment let us show with some examples that the second stage of our method is in fact performing local contrast enhancement.

As the first example we consider a simple image with gray bands, on the top left of Fig. 4, with its corresponding profile of a scan line on the bottom left of the figure. When we apply to it the first stage of our algorithm we obtain the image on the top middle: there is no Mach band phenomena, as we can verify by looking at the bottom middle profile, where the order of the heights of the bands is preserved. When we apply the second stage of our algorithm we obtain the image on the top right, and now we see the Mach band phenomena appear, as we can check by looking at the under- and over-shoots in the profile of the image in the bottom right.

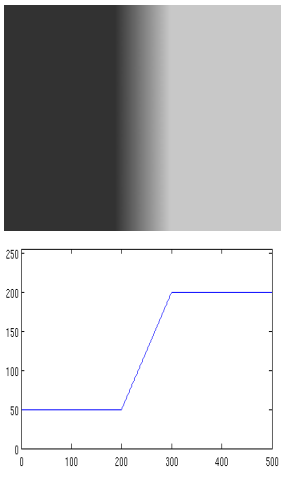

(a)

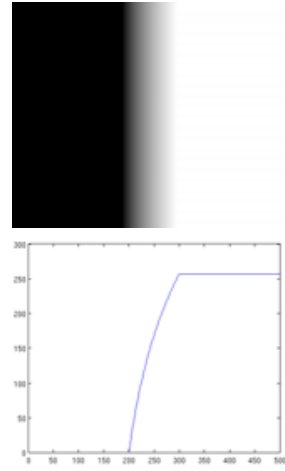

(b)

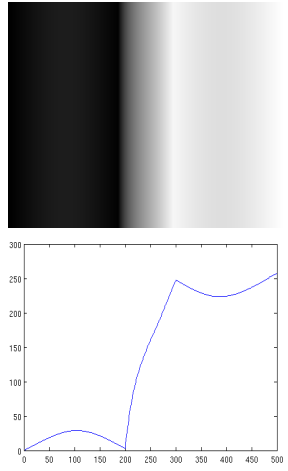

(c)
Figure 4. Top row: (a) original image, (b) after first stage of our TMO, (c) final output of our TMO. Bottom row: corresponding profiles of scan lines.

As a second example we consider the picture in Fig. 5 where Fig. 5(a) is the LDR output of the first stage of our TMO, Fig. 5 (b) is the output of the second stage (the final output) of our algorithm. While the overall colors and contrast have not changed, the final output shows considerably more detail on the stone arch and columns. This qualitative assertion can be quantified using a metric validated by psychophysical experiments in the work by Aydin et al. [42]. This metric will be explained in more detail in section $\mathrm{V}$, but for now let us just say that the metric marks as green those pixels with loss of visible contrast. Fig. 5 (c) shows the error for the first stage of our method and Fig. 5)(d) shows the error for the final output. We can see that, when we apply the second stage, the loss of contrast is reduced appreciably (numerically, the area of pixels with loss of contrast is reduced by almost fifty percent.)

\section{IMPLEMENTATION}

Let us start this section on the implementation details of our algorithm by saying that we deal with each of the three color 


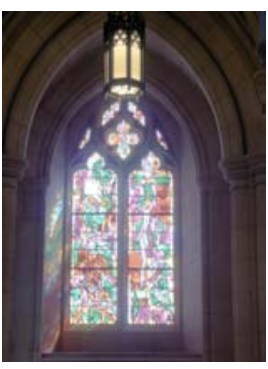

(a)

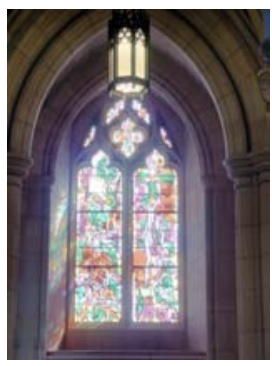

(b)

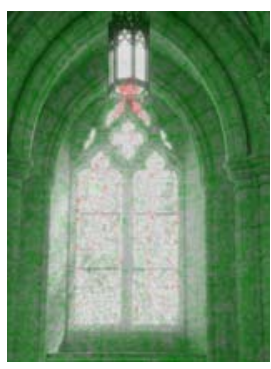

(c)

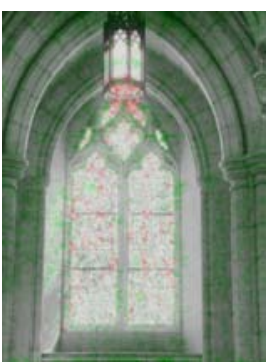

(d)

Figure 5. (a) Output of first stage (b) Output of second stage (c) Visualization of error of first stage (d) Visualization of error of final output. Error computed with the metric of Aydin et al. [42].

channels separately (most TM methods consider the luminance channel and do not operate separately on each color channel, see [2]). For the experiments we have used the original $R, G$, and $B$ channels of the images. Hence, in what follows, when we talk about radiance values, averages, etc., we are referring to real-valued images corresponding to one color channel, not vector valued images corresponding to three channels simultaneously.

As we mentioned in section III, several works in neuroscience point out that cones change their sensitivity in proportion to the light level they receive in their same waveband (see [29], [30]), that is why we have chosen to compute the background intensity and semisaturation constants independently for each channel, i.e. not based on the luminance of the image, as it is usually done.

For each channel we compute the background intensity $\mathcal{I}_{b}$ as the geometric average between the arithmetic mean and the median of the radiance values: $\mathcal{I}_{b}=$ median $^{0.5}$ mean $^{0.5}$. We introduce one parameter, $\rho$, that lets the user specify the overall brightness of the final output, so our estimated background intensity becomes: $\mathcal{I}_{b} 10^{-\rho}$. The default value for $\rho$ is zero; $\rho>0$ increases the darkness of the output, $\rho<0$ increases its brightness. The effect of varying $\rho$ can be observed in Fig. 6 first row.

Valeton and van Norren [28] show how the Naka-Rushton curves shift to the right as $\mathcal{I}_{b}$ increases, and how to compute the corresponding semisaturation constant $\mathcal{I}_{s}$ from the background intensity. For the latter we linearly interpolated their data obtaining the function ${ }^{2} \log _{10} \mathcal{I}_{s}=\log _{10}\left(\mathcal{I}_{b}\right)-0.37(4+$ $\left.\log _{10}\left(\mathcal{I}_{b}\right)-\rho\right)+1.9$.

The semi-saturation value is, by definition, the center value in the 4-order operative range of the cones, so the inflexion point $\mathcal{I}_{M}$ in eq. (6) where we switch from Weber-Fechner to Naka-Rushton as explained in section II is computed as $\mathcal{I}_{M}=\mathcal{I}_{s} 10^{2}$. The values for the Weber-fractions $k$ in eq. (6) are taken from experimental data in Wyszecki and Stiles [32]: $\hat{k}=\frac{100}{1.85}$ for "red" and "green" cones, $\hat{k}=\frac{100}{8.7}$ for "blue" cones. Following Valeton and van Norren [28] we take $n=0.74$. The value for the constant $s_{0}$ is computed so that the curve $c$ is continuous at $\mathcal{I}_{M}$. The value for $m$ is chosen as $m=\mathcal{I}_{s} 10^{-1.2}$, following graphs in Wyszecki and Stiles [32].

\footnotetext{
${ }^{2}$ The data in Valeton and van Norren [28] is in trolands, while calibrated HDR data is in $\frac{c d}{m^{2}}$. In log-scale this accounts to a shift that we introduced as the constant 4 which is added to $\log _{10}\left(\mathcal{I}_{b}\right)$.
}

After we apply eq. (6) to the input HDR image, we normalize the result (linearly scaling it so that its range becomes $[0,1])$. Denoting by $I_{0}$ any of the color channels of this image, we now look for the image $\tilde{I}$ minimizing eq. (8) through the gradient descent method by iterating the following procedure until convergence, as proposed by Palma-Amestoy et al. [39]:

$$
I^{n+1}(x)=\frac{I^{n}(x)+\Delta t\left(\mu_{0}+I_{0}(x)+\frac{1}{2} R_{I^{n}}(x)\right)}{1+2 \Delta t},
$$

where $\Delta t=0.2, \mu_{0}$ is the mean of $I_{0}$ and

$$
R_{I^{n}}(x)=\int_{\Omega} w(x, y) \operatorname{sign}\left(I^{n}(x)-I^{n}(y)\right) d y,
$$

with $w$ being a normalized 2D Gaussian kernel of effective radius $\sigma$. We choose to set $\sigma$ as $30 \%$ of the number of rows or columns of the image (whichever is greater), and if we wanted to modify its value we would see an effect on the local contrast properties of the output, as it can be observed in Fig. 6 second row.

It is important to note that, since the values for $\rho$ and $\sigma$ influence the overall brightness of the output and its local contrast properties, they could be optimized for any particular display: we could use a quality measure like that of Aydin et al. [42] (discussed in the following section) in order to find the values of $\rho$ and $\sigma$ that, for a given display, produce less error.

The iteration process stops when the average difference between $I^{n}$ and $I^{n-1}$ is less than $0.5 \%$. Then we linearly scale $I^{n}$ so that its maximum is 1 and its minimum is 0 , obtaining the final output $\tilde{I}$. The rationale for this scaling is to make use of all the available range in the LDR image, but we are aware that this potentially results in contrast stretching, independently in each color channel, possibly leading to color artifacts: we have not observed such problems in our experiments (see next section) but in any case we want to remark that this last scaling is not at all an essential part of our procedure and in fact it may be omitted. Eq. (9) has complexity of order $O\left(N^{2}\right)$, where $N$ is the total number of pixels, but it can be reduced to $O(N \log N)$ with the numerical approximation technique proposed by Bertalmío et al. [38]. Our implementation is written in $\mathrm{C}++$ and runs under Linux on a computer with $3 \mathrm{~GB}$ RAM and two $2.8 \mathrm{GHz}$ processors. For a $1024 \times 768$ image the first stage of our algorithm takes 1.13 seconds and the second stage takes 10.8 seconds. 

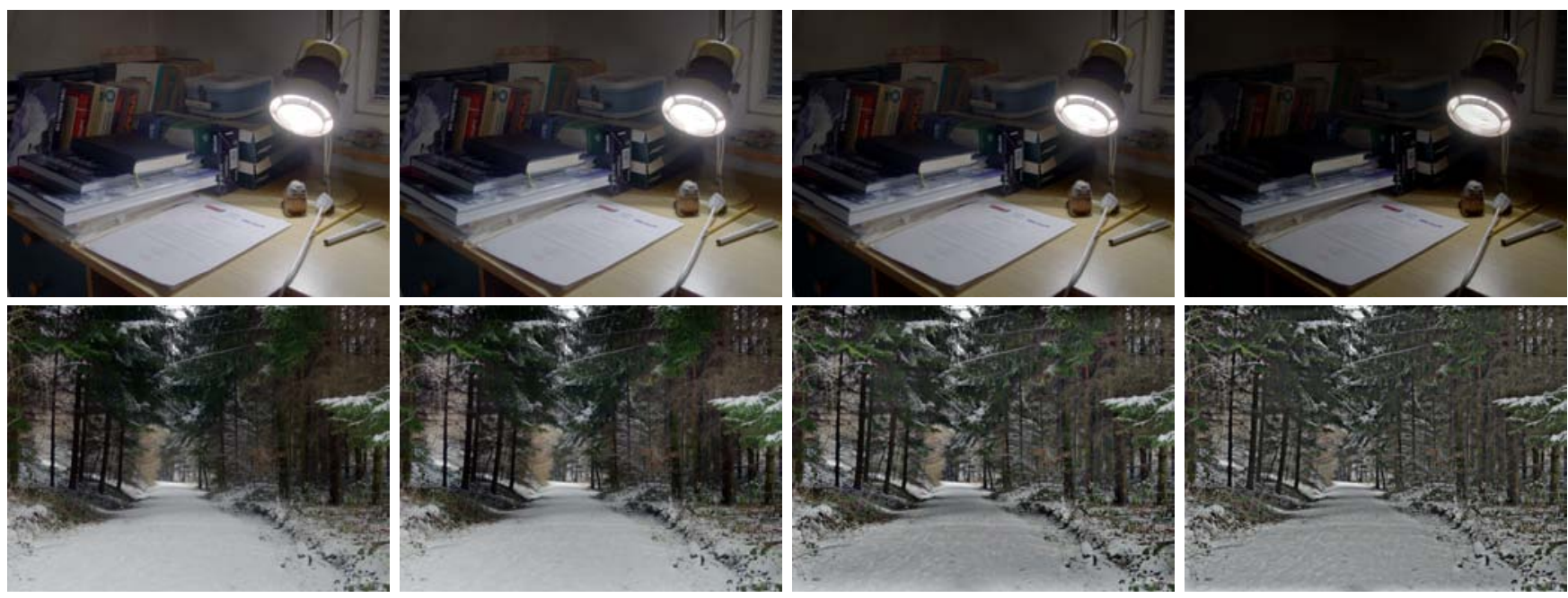

Figure 6. On the first row, effect of varying $\rho$. From left to right: $\rho=-1,0,1,2$. Second row, effect of varying $\sigma$. From left to right: $\sigma=50 \%, 20 \%, 4 \%, 2 \%$ of the number of rows or columns or the image, whichever is greater.

\section{RESULTS AND COMPARISONS}

As stated in the Introduction, a good TMO should reproduce realistically the color sensation and detail visibility of the original scene. Thus, in order to evaluate the performance of our algorithm, we should quantitatively measure its ability to reproduce these two features. We must point out that our TMO does not take into account the changes in color (hue, saturation, brightness) and contrast (visual acuity) that happen at very low luminance levels: for this we could process the output of our algorithm with a method such as the one proposed by Haro et al. [43].

Regarding color sensation, presently there are two color models for HDR images which are based on psychophysical experiments: Mantiuk et al. [44] propose color-correction formulas that can be applied to existing TMOs, and Kim et al. [45] propose a color appearance model after conducting experiments covering most of the dynamic range of the HVS. However, these methods are not yet able to, given an HDR image and its LDR mapping performed by a given TMO, output a numerical value that measures how well the colors of the tone-mapped image match the sensation produced by the HDR image. Qualitatively, we can say we have observed that the outputs of our algorithm do not show color artifacts and that these images seem to us natural-looking, in terms of color saturation and hue: Fig. 12, Fig. 11, and also Figs. 3, 5 . 6.

With respect to detail visibility or contrast, several methods can be found in the literature. Drago et al. [46], Yoshida et al. [47] and Kuang et al. [48] ran psychological experiments asking subjects to evaluate the similarity between a real-world scene and a tone-mapped image of that scene. Ledda et al. [49] also presented a methodology based on psychological judgments, but they substituted the real-world scene with a linearly mapped HDR image seen on a HDR screen. Cadík et al. [50] propose a basic set of attributes to evaluate tone-mapped images, perform experiments where users rank images according to those attributes, and use those statistics to propose a measure of the overall image quality as a function of said attributes. Recently, in a remarkable work, Aydin et al. [42] proposed a quantitative measure for tone mapping quality based on psychophysical experiments. Given an HDR reference and its LDR tone-mapping, this method first estimates the luminance values produced by the LDR image shown in a specific display ${ }^{3}$. Then, it compares the perception a viewer would have of both scenes relying on psychophysical data. The output is an image of the same size as the original called distortion map where they use color coding to represent the contrast distortion error for each pixel: gray means no error, green represents loss of visible contrast, blue represents amplification of contrast (i.e. we can see in the LDR image detail we should not be able to see) and red represents contrast reversal (contrast visible both on the HDR and LDR images, but with different polarity.) The saturation of each color indicates the magnitude.

In Fig. 7 we compare the first stage of our algorithm with three global TMOs: we can see that our results exhibit less contrast distortion than the ones obtained with the other methods. Normally the second stage improves on the results of the first one by enhancing local contrast (and removing color cast), as it was shown in Fig. 5. but Fig. 8 shows that, when the image is too dark, the output of the first stage of our algorithm is practically free of errors while the local contrast enhancement stage produces lots of amplification errors, i.e. we are able to see more detail than what would actually be possible, even if there are no halo artifacts. These errors could be ameliorated by selecting an even higher value of $\sigma$ for this image (we used $\sigma=30 \%$ ). Fig. 8 shows the importance of having an objective quality metric to measure TM results, since from a subjective standpoint one would be led to prefer the result of Fig. 8(b).

In Fig. 12 we show the results for our algorithm and for several state of the art methods. We can see that, for both examples shown in the figure, our algorithm exhibits less

\footnotetext{
${ }^{3}$ For all the examples in this paper where we have used this metric we are assuming a generic display with $\gamma=2.2,30$ pixels per degree and viewing
} distance of $0.5 \mathrm{~m}$. 
contrast distortion. For the image set consisting of the images shown in Figs. 11 and 12 we have run our algorithm and several others: Drago et al. [46], Fattal et al. [20], Mantiuk et al. [21], Pattanaik et al. [8], Reinhard et al. [14] and Reinhard and Devlin [9]. From the distortion maps provided by the method of Aydin et al. [42] we extracted the error value for each pixel and computed the following averages over our image set: total error percentage, reversal error percentage (red), loss of contrast error percentage (green), contrast amplification error percentage (blue). Note that the measure proposed by Aydin et al. only assigns one type of error to each pixel, the type of error which is predominant at that location. The numerical results are summarized ${ }^{4}$ in Fig. 10 It is important to note that these results would be different for a different display and, as we suggested in the previous section, we could use this quality metric in order to find the values of $\rho$ and $\sigma$ of our method that, for a given display, produce less error. We have run our algorithm in two modes: with a fixed $\rho=0$ (the default value) and with variable $\rho$ (chosen different for each image so as to minimize its error), and always with fixed $\sigma=30 \%$.

We can see that the results, although better for variable $\rho$, do not improve much over the results obtained with the default value of $\rho$. In both cases, though, and for our image set, our method produces considerably less total contrast distortion than the other methods in the state of the art, and the minimum reverse and amplification error of all methods considered. We must point out that for comparison with the state of the art we are using the algorithms provided in the PFSTool $5^{5}$ package, with the default or suggested parameters for every method. It is interesting to note that the first stage of our method, given by eq. 6 and therefore a global procedure, performs better in terms of total contrast distortion than the global TMOs of Drago et al. [46], Pattanaik et al. [8], and Reinhard and Devlin [9] (see last row of Fig. 10p. Remarkably, the total error for this first stage is also a bit lower than the total error of our proposed TMO (see next-to-last column of the table). But more careful inspection shows that, while the second stage is performing local contrast enhancement and hence the error associated to loss of contrast is highly reduced in the final output, as expected, the amplification error increases by an amount similar to the decrease in the loss of contrast error, which explains why the total errors of the first stage and the final output of our proposed method are comparable. Should we then discard the second stage and propose a global TMO only? We believe that the second stage is essential, because the first stage is not able to reproduce basic perceptual phenomena like the Mach bands effect, as we argued in section III] but also because the amplification error, which is the only type of error that increases considerably with the second stage of our TMO, can be reduced by an appropriate selection of the kernel width $\sigma$, as we mentioned above regarding Fig. 8 .

Also, we point out that the reversal error is low for our algorithm. If we consider halos as an inversion of the order of

${ }^{4}$ For the complete numerical figures please go to http://www.gpi.upf.edu/ static/sira/Sira_Ferradans/Tone_Mapping

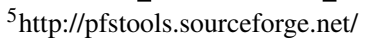

the level lines, then the first stage of our method cannot create halos (given that it is a non-decreasing global function), but the second stage can. However, this second stage was proven by Bertalmío et al. [38] to produce only a slight inversion of the level lines, the amount of said inversion depending on the parameter $\sigma$ (see the second row of Fig. 6): as $\sigma$ increases this effect disappears. All the results presented in this article were obtained with the rather high value of $\sigma=30 \%$, thus the reversal error is low.

We have also computed statistics for the number of pixels with error, shown in Fig. 9 for each of the images in our set and for all the TMOs considered. Our method has a slightly poorer performance with respect to the loss of contrast; but in the reversal, amplification and total errors it appears to be consistently better than the other methods, both with fixed and variable $\rho$.

\begin{tabular}{|c|c|c|c|c|}
\hline Methods & Reverse & Loss & Amplification & Total \\
\hline Drago et al. [46] & 4 & 4.9 & 37.32 & 46.21 \\
\hline Fattal et al. [20] & 11.58 & 0.64 & 43.05 & 55.27 \\
\hline Mantiuk et al. [21] & 1.39 & 10.56 & 26.98 & 38.93 \\
\hline Pattanaik et al. [8] & 6.41 & 6.65 & 23 & 36.06 \\
\hline Reinhard et al. [14] & 5.5 & 4.92 & 38.06 & 48.48 \\
\hline $\begin{array}{c}\text { Reinhard and } \\
\text { Devlin [9] }\end{array}$ & 5.81 & 3.71 & 34.43 & 43.95 \\
\hline Our TMO, $\rho=0$ & 0.74 & 9.23 & 16.61 & 26.58 \\
\hline $\begin{array}{c}\text { Our TMO, } \\
\text { selecting } \rho\end{array}$ & 1.10 & 7.28 & 14.92 & 23.30 \\
\hline $\begin{array}{c}\text { Only 1st stage, eq. 6 } \\
\text { selecting } \rho\end{array}$ & 0.61 & 12.65 & 5.52 & 18.78 \\
\hline
\end{tabular}

Figure 10. Error percentages computed with Aydin et al. [42], averaged over our image data set, for several TMOs (see text.)

\section{CONCLUSIONS AND PERSPECTIVES}

We have proposed a tone mapping operator inspired by basic perceptual properties of human vision: visual adaptation and local contrast enhancement. The results look realistic in terms of color, in the authors' opinion. In terms of contrast rendition they outperform the state of the art, a claim supported by quantitative results obtained with a metric validated by psychophysical experiments. We show numerically that our method produces less error in terms of contrast distortion and area of pixels with distortion.

As a possibility for improvement we could look into more precise t.v.i. models instead of the Weber-Fechner law. Also, the proposed method has two parameters that control the overall brightness and the contrast of the final image, which are directly related to specific display properties: it would be interesting to investigate how to automatically select these parameters so as to minimize the error metric of Aydin et al. [42], for each given display. Furthermore, we would like to combine our method with this metric and develop an iterative procedure that at each step and for each pixel performs the sort of contrast modification (enhancement, reduction, or none) dictated by the distortion map for that pixel, hoping to 


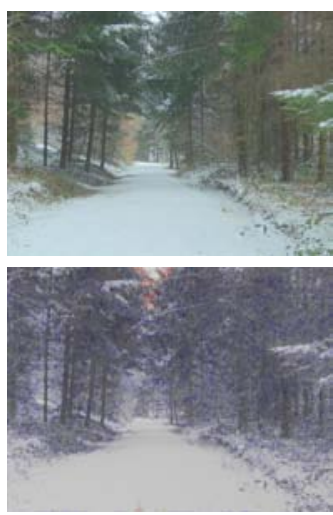

(a)
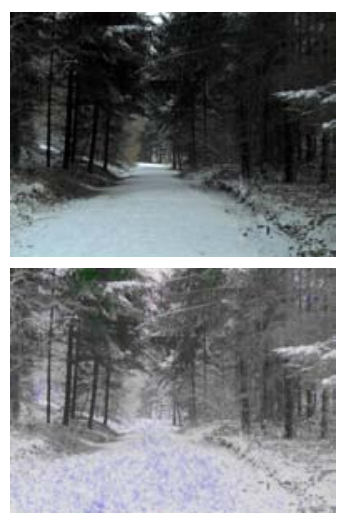

(b)
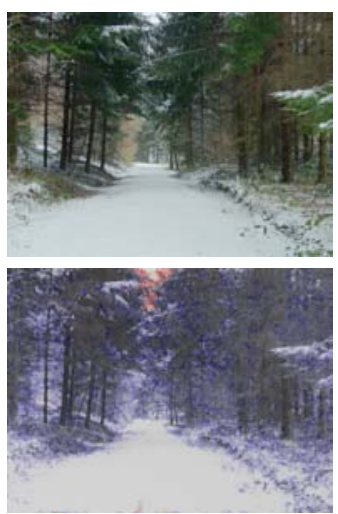

(c)
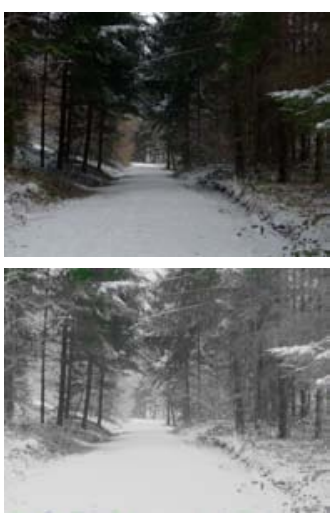

(d)

Figure 7. Comparison with global TMOs. TM results (top row) and distortion maps (bottom row) for: (a) Drago et al. [46] (b) Pattanaik et al. [8] (c) Reinhard and Devlin [9] (d) first stage of our TMO.

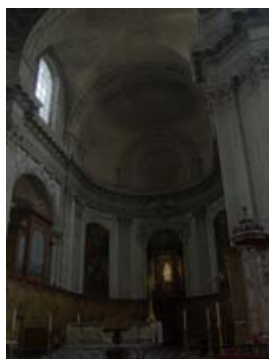

(a)

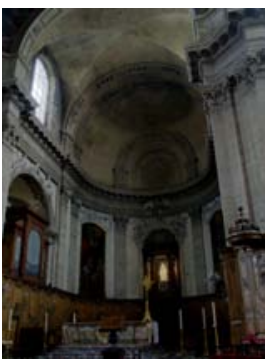

(b)

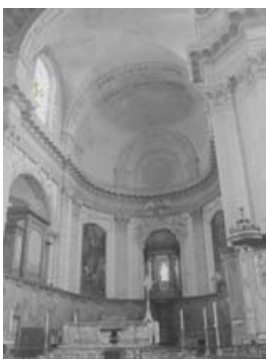

(c)

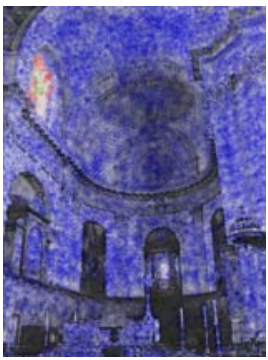

(d)

Figure 8. (a) Output of the first stage. (b) Output of the second stage. (c) Visualization of the first stage error. (d) Visualization of the final output error. Error computed with the metric of Aydin et al. [42].
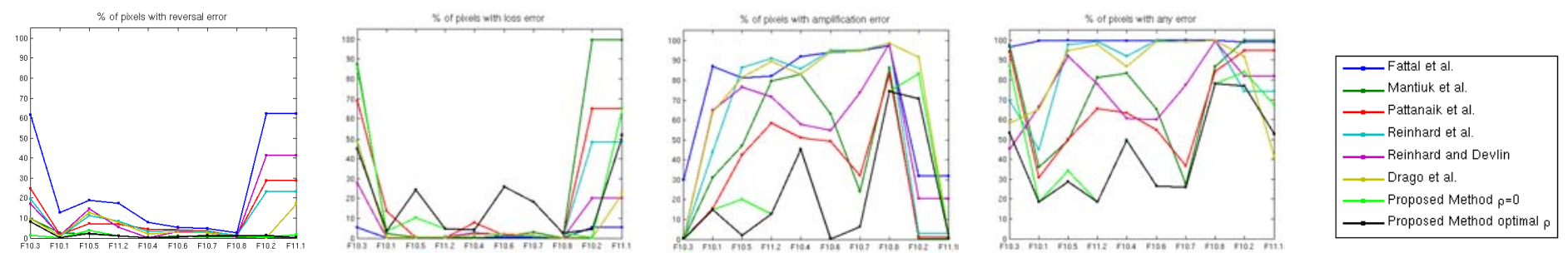

Figure 9. Percentage of pixels with errors, for our image set and for the methods considered. Horizontal axis: images in Figs. 11 and 12

converge to an image with an overall error much closer to zero than what we can achieve now.

\section{ACKNOWLEDGMENTS}

The authors are extremely grateful to T. Aydin, who has provided us with the quality metric results, and to the reviewers, which have contributed a great deal to the improvement of the manuscript and the proposed method. M. Bertalmío and V. Caselles acknowledge partial support by PNPGC project, reference MTM2006-14836. We acknowledge partial support by by GRC reference 2009 SGR 773 funded by the Generalitat de Catalunya. V. Caselles also acknowledges "ICREA Acadèmia" prize by the Generalitat de Catalunya. E. Provenzi acknowledges the Ramón y Cajal fellowship by Ministerio de Ciencia y Tecnología de España.

\section{REFERENCES}

[1] T. Kunkel and E. Reinhard, "A reassessment of the simultaneous dynamic range of the human visual system," in APGV '10: Proceedings of the 7th Symposium on Applied Perception in Graphics and Visualization. New York, NY, USA: ACM, 2010, pp. 17-24.

[2] E. Reinhard, G. Ward, S. Pattanaik, and P. Debevec, High Dynamic Range Imaging, Acquisition, Display, And Image-Based Lighting. Morgan Kaufmann Ed., 2005.

[3] S. Stevens, "On the psychophysical law," Psychological Review, vol. 64, pp. 153-181, 1957.

[4] J. Tumblin and H. Rushmeier, "Tone reproduction for realistic images," IEEE Computer Graphics and Applications, pp. 42-48, 1993.

[5] K. Chiu, M. Herf, P. Shirley, S. Swamy, C. Wang, and K. Zimmerman, "Spatially nonuniform scaling functions for high contrast images," in Proceedings of Graphics Interface 93. Morgan Kaufmann, 1993, pp. 245-253.

[6] G. Ward, "A contrast-based scalefactor for luminance display," Graphics gems IV, pp. 415-421, 1994.

[7] C. Schlick, "Quantization techniques for visualization of high dynamic range pictures," in Proceedings of the 5th Eurographics Workshop on Rendering Workshop. Springer Verlag, 1994, pp. 7-20.

[8] S. Pattanaik, J. Tumblin, H. Yee, and D. Greenberg, "Time-dependent 

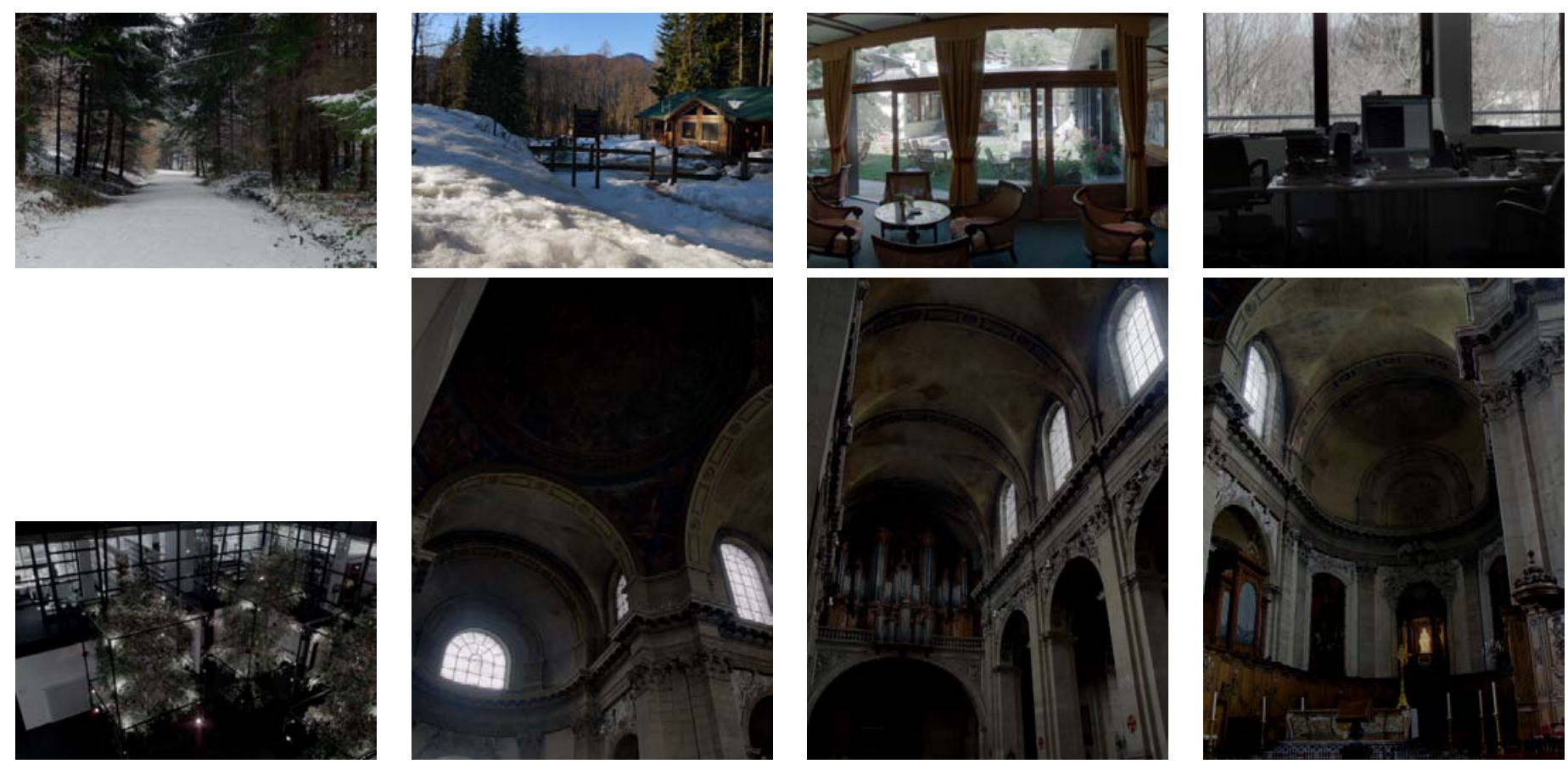

Figure 11. Results of our method for several images obtained from the MPI database, see http://www.mpi-inf.mpg.de/resources/hdr/gallery.html for further details.
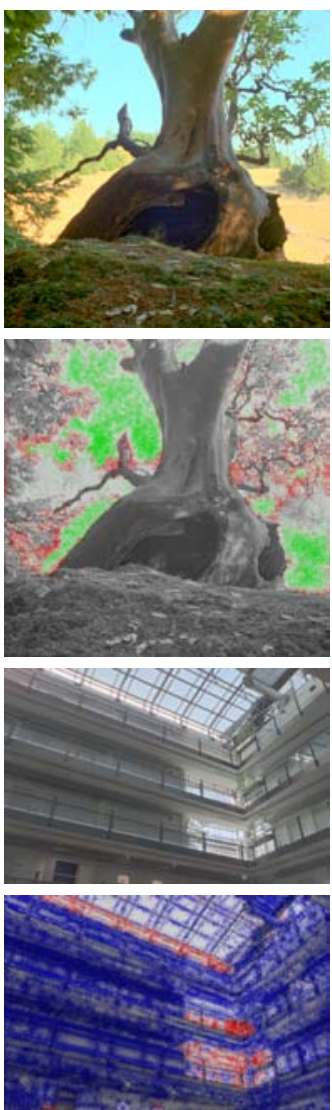

(a)
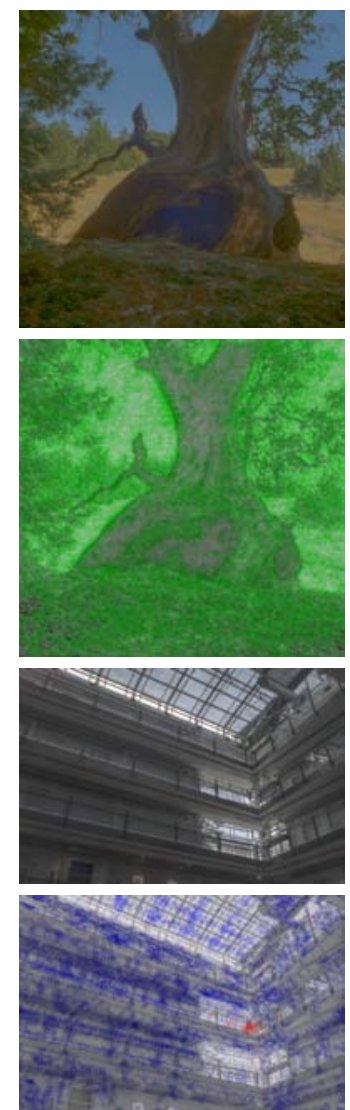

(b)
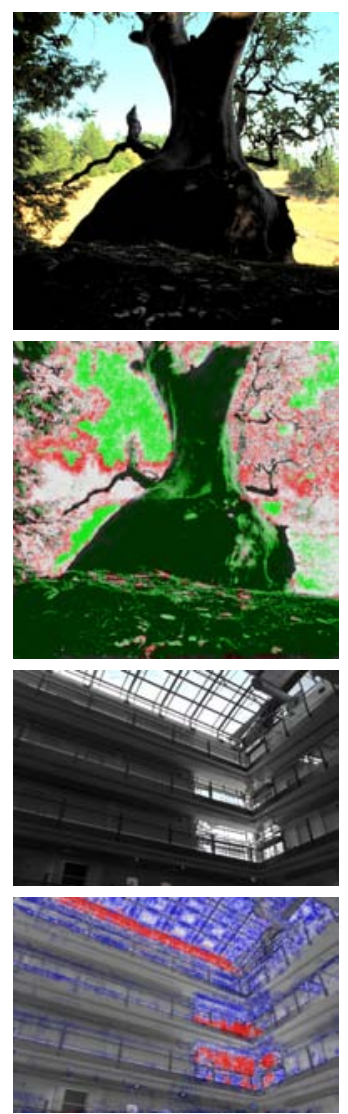

(c)
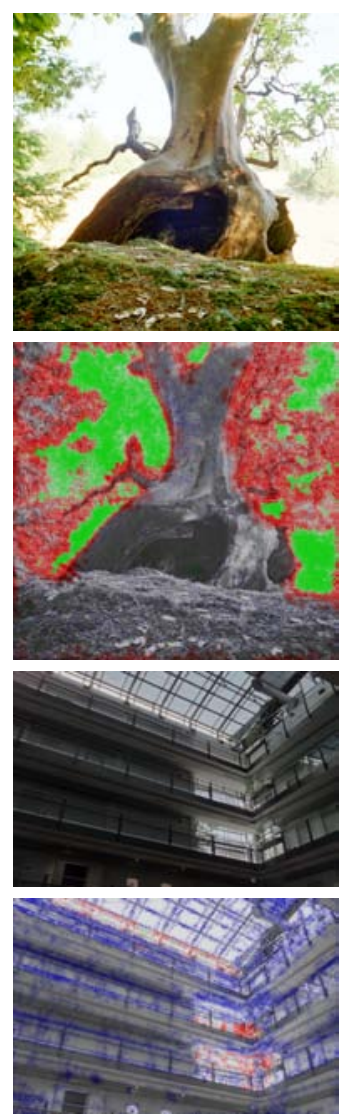

(d)
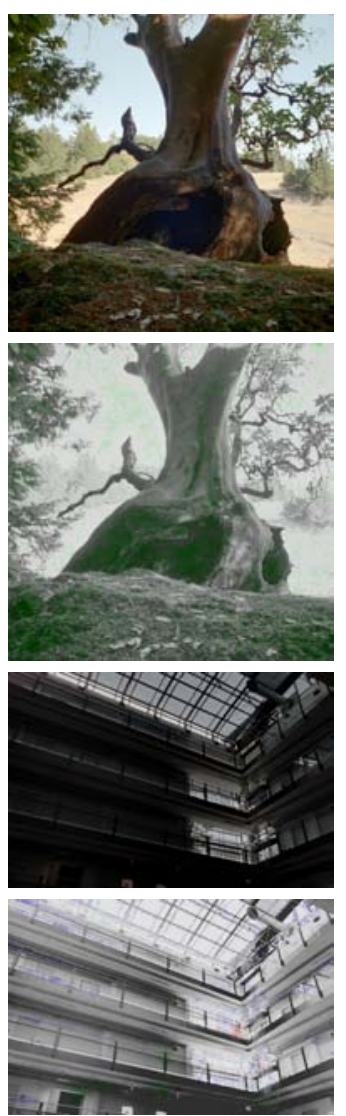

(e)

Figure 12. TM results (rows 1 and 3) and distortion maps (rows 2 and 4) for these TMOs: (a) Drago et al. [46] (b) Mantiuk et al. [21] (c) Pattanaik et al [8] (d)Reinhard and Devlin [9] (e) the proposed method. Original image from row 1 courtesy of ILM. Original image from row 3 courtesy of R. Mantiuk.

visual adaptation for fast realistic image display," in Proceedings of SIGGRAPH, 2000, pp. 47-54.
[9] E. Reinhard and K. Devlin, "Dynamic range reduction inspired by photoreceptor physiology," IEEE Trans. on Visualization and Computer 
Graphics, vol. 11(1), pp. 13-24, 2005.

[10] J. Kuang, G. Johnson, and M. Fairchild, "iCAM06: A refined image appearance model for HDR image rendering," J. Vis. Commun. Image R., vol. 18, pp. 406-414, 2007.

[11] J. Ferwerda, S. Pattanaik, P. Shirley, and D. Greenberg, "A model of visual adaptation for realistic image synthesis," in Proceedings of SIGGRAPH 96, Computer Graphics Proceedings. Addison Wesley, 1996, pp. 249-258.

[12] G. Ward, H. Rushmeier, and C. Piatko, "A visibility matching tone reproduction operator for high dynamic range scenes," IEEE Transactions on Visualization and Computer Graphics, vol. 3, pp. 291-306, 1997.

[13] M. Ashikhmin, "A tone mapping algorithm for high contrast images," in Eurographics Workshop on Rendering. P. Debevec and S. Gibson Eds., 2002, pp. 1-11.

[14] E. Reinhard, M. Stark, P. Shirley, and J. Ferwerda, "Photographic tone reproduction for digital images," ACM Trans. on Graph., vol. 21, pp. 267-276, 2002.

[15] D. Tamburrino, D. Alleysson, L. Meylan, and S. Susstrunk, "Digital camera workflow for high dynamic range images using a model of Retinal processing," in Proceedings SPIE, vol. 6817, 2008.

[16] D. Jobson, Z. Rahman, and G. Woodell, "A multiscale Retinex for bridging the gap between color images and the human observation of scenes," IEEE Transactions on image processing, vol. 6, no. 7, pp. 965976, july 1997.

[17] G. Krawczyk, K. Myszkowski, and H. Seidel, "Lightness perception in tone reproduction for high dynamic range images," pp. 635-645, 2005.

[18] J. Tumblin and G. Turk, "Lcis: A boundary hierarchy for detailpreserving contrast reduction," in SIGGRAPH: Conference Proceedings, 1999, pp. 83-90.

[19] F. Durand and J. Dorsey, "Fast bilateral filtering for the display of highdynamic-range images." SIGGRAPH 2002, Proceedings of the 29th annual conference on Computer graphics and interactive techniques, 2002, pp. 257-266.

[20] R. Fattal, D. Lischinski, and M. Werman, "Gradient domain high dynamic range compression," in ACM Trans. Graphics, vol. 21 (3), 2002, pp. 249-256.

[21] R. Mantiuk, K. Myszkowski, and H. Seidel, "A perceptual framework for contrast processing of high dynamic range images," ACM Transactions on Applied Perception (TAP), vol. 3 (3), pp. 286-308, 2006.

[22] A. Pardo and G. Sapiro, "Visualization of high dynamic range images," IEEE Transactions on Image Processing, vol. 12, no. 6, pp. 639-647, 2003.

[23] D. Lischinski, Z. Farbman, M. Uyttendaele, and R. Szeliski, "Interactive local adjustment of tonal values," in SIGGRAPH '06: ACM SIGGRAPH 2006 Papers. New York, NY, USA: ACM, 2006, pp. 646-653.

[24] F. Dunn, M. Lankheet, and F. Rieke, "Light adaptation in cone vision involves switching between receptor and post-receptor sites," Nature, vol. 449, no. 7162, pp. 603-606, 2007.

[25] D. Hubel, Eye, Brain, and Vision. Scientific American Library, 1995.

[26] R. Shapley and C. Enroth-Cugell, Visual adaptation and retinal gain controls, 1984, vol. 3, pp. 263-346.

[27] J. Keener and J. Sneyd, Mathematical Physiology. Springer, 2008.

[28] J. Valeton and D. van Norren, "Light adaptation of primate cones: an analysis based on extracellular data," Vision Research, vol. 23, no. 12, pp. $1539-1547,1983$.

[29] G. Boynton, "Color vision: How the cortex represents color," Current Biology, vol. 12, no. 24, pp. R838-R840, 2002.

[30] B. Wade, Wandell, "Chromatic light adaptation measured using functional magnetic resonance imaging," Journal of Neuroscience, vol. 22, pp. 8148-8157, 2002.

[31] S. Palmer, Vision Science: Photons to Phenomenology. The MIT Press, 1999.

[32] G. Wyszecki and W. S. Stiles, Color science: Concepts and methods, quantitative data and formulas. John Wiley \& Sons, 1982.

[33] P. Irawan, J. A. Ferwerda, and S. R. Marschner, "Perceptually based tone mapping of high dynamic range image streams," in Rendering Techniques, 2005, pp. 231-242.

[34] S. Shevell, "Saturation in human cones," Vision Research, vol. 17, no. 3, pp. 427-434, 1977.

[35] S. Stevens, "To honor Fechner and repeal his law," Science, vol. 133, pp. 80-133, 1961.

[36] N. Macmillan and C. Creelman, Detection theory: A user's guide. Lawrence Erlbaum, 2005

[37] E. Land and J. McCann, "Lightness and Retinex theory," Journal of the Optical Society of America, vol. 61, no. 1, pp. 1-11, Jan. 1971.
[38] M. Bertalmío, V. Caselles, E. Provenzi, and A. Rizzi, "Perceptual color correction through variational techniques," IEEE Trans. on Image Processing, vol. 16, pp. 1058-1072, 2007.

[39] R. Palma-Amestoy, E. Provenzi, M. Bertalmío, and V. Caselles, "A perceptually inspired variational framework for color enhancement," IEEE Transactions on Pattern Analysis and Machine Intelligence, vol. 31, no. 3, pp. 458-474, 2009.

[40] M. Bertalmío, V. Caselles, and E. Provenzi, "Issues about the Retinex theory and contrast enhancement," to appear in International Journal of Computer Vision, 2009.

[41] E. Provenzi, L. De Carli, A. Rizzi, and D. Marini, "Mathematical definition and analysis of the Retinex algorithm," Journal of the Optical Society of America A, vol. 22, no. 12, pp. 2613-2621, Dec. 2005.

[42] T. Aydin, R. Mantiuk, K. Myszkowski, and H. Seidel, "Dynamic range independent image quality assessment," in ACM SIGGRAPH 2008 papers, 2008, pp. 1-10.

[43] G. Haro, M. Bertalmío, and V. Caselles, "Visual acuity in day for night," International Journal of Computer Vision, vol. 69, no. 1, pp. 109-117, 2006.

[44] R. Mantiuk, R. Mantiuk, A. Tomaszewska, and W. Heidrich, "Color correction for tone mapping," Computer Graphics Forum (Proc. EUROGRAPHICS), vol. 28, no. 3, pp. 193-202, 2009.

[45] M. Kim, T. Weyrich, and J. Kautz, "Modeling human color perception under extended luminance levels," ACM Transactions on Graphics (TOG), vol. 28, no. 3, p. 27, 2009.

[46] F. Drago, K. Myszkowski, T. Annen, and N. Chiba, "Adaptive logarithmic mapping for displaying high contrast scenes," Computer Graphics Forum, vol. 22, pp. 419-426, 2003.

[47] A. Yoshida, V. Blanz, K. Myszkowski, and H. Seidel, "Perceptual evaluation of tone mapping operators with real-world scenes," in Human Vision and Electronic Imaging 10. SPIE, 2005, pp. 192-203.

[48] J. Kuang, H. Yamaguchi, C. Liu, G. M. Johnson, and M. Fairchild, "Evaluating HDR rendering algorithms," ACM Trans. Appl. Percept., vol. 4, no. 2, p. 9, 2007.

[49] P. Ledda, A. Chalmers, T. Troscianko, and S. H., "Evaluation of tone mapping operators using a high dynamic range display," Proceedings ACM Transactions on Graphics, vol. 24, pp. 640-648, 2005.

[50] M. Cadík, M. Wimmer, L. Neumann, and A. Artusi, "Evaluation of HDR tone mapping methods using essential perceptual attributes," Computers \& Graphics, vol. 32, no. 3, pp. 330-349, 2008.

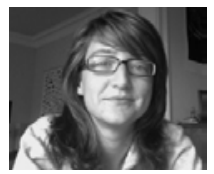

Sira Ferradans received the Computer Science degree from the Universitat Pompeu Fabra (UPF, Barcelona, Spain) in 2005 and her M.Sc. in computer vision from the Universitat Autonoma de Barcelona in 2006. Currently, she is pursuing her Ph.D. in Image Processing at the UPF under the supervision of Marcelo Bertalmío and Vicent Caselles. Her research interests include image processing, computational photography and its applications.

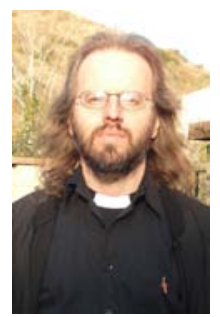

Marcelo Bertalmío received his B.Sc. and M.Sc. degrees from Universidad de la República, Uruguay, in 1996 and 1998 respectively, and his Ph.D. from the University of Minnesota 
in 2001. He is an associate professor at Universitat Pompeu Fabra, Spain, since 2006. He is interested in image processing and computer vision and their applications to the movie industry.

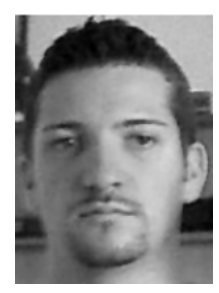

Edoardo Provenzi received the master degree in Physics at University of Milano (Italy) in 2000 and the PhD in Mathematics and applications at University of Genova (Italy) in 2004. He currently has a post-doc position at Universitat Pompeu Fabra of Barcelona (Spain) developing his research on mathematical models of color vision and imaging.

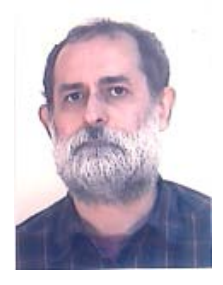

Vicent Caselles received the Licenciatura and $\mathrm{Ph}$. D. degrees in mathematics from Valencia University, Spain, in 1982 and 1985, respectively. Currently, he is professor at the Pompeu Fabra University (Barcelona). $\mathrm{He}$ is an associate member of IEEE. His research interests include image processing, computer vision, and the applications of geometry and partial differential equations to both previous fields. 